\title{
DUSTY COMETARY ATMOSPHERES
}

\author{
Tamás I. Gombosi \\ Space Physics Research Laboratory, The University of Michigan, Ann Arbor, MI \\ 48109, U.S.A. and Central Research Institute for Physics, Budapest P.O.B 49, \\ Hungary 1525
}

\begin{abstract}
This paper summarizes our present understanding of the physical processes controlling the dust and gas production of cometary nuclei and the evolution of the dusty gas flow in the inner coma. Special emphasis is being made to compile a self-consistent set of governing equations describing the accelerating dusty gas flow in a cometary atmosphere.

\section{INTRODUCTION}

The cometary atmosphere is a unique phenomenon in the solar system. Due to its negligible gravity the tiny nucleus of only a few kilometers produces a highly variable, extensive dusty atmosphere of dimensions ranging from $10^{4} \mathrm{~km}$ to $10^{5} \mathrm{~km}$. The large spatial extension and continual expansion of a cometary atmosphere provides a convenient tool for studying the time history of atmospheric processes.

One of the most important features influencing cometary dynamics is the "retarded" nature of gas and dust production. The radiation reaching the surface and supplying energy for sublimation must first penetrate an extensive, absorbing dusty atmosphere. Any change in the gas and dust production alters the optical characteristics of the atmosphere, thus affecting the radiation reaching the surface at a later time.

Recently we had the first opportunity to investigate a cometary environment from close range and to collect a new and extremely valuable set of information about cometary atmospheres. This paper briefly overviews some of the present atmospheric models in light of the new spacecraft observations. For a detailed summary of these models we refer to recent comprehensive reviews $/ 1,2 /$.
\end{abstract}

\section{GAS AND DUST PRODUCTION}

Our present understanding of cometary nuclei is based on Whipple's "dirty iceball" idea $/ 3 /$ according to which the nucleus consists of a mixture of frozen volatiles and non-volatile dust. The most recent observations, together with other evidence, led Weissman to postulate that the nucleus is a primordial rubble pile of weakly-bound, small icy conglomerates $/ 4 /$. Very recently the first images of the comet Halley nucleus inspired Gombosi and Houpis to propose an icy-glue model, suggesting that the nucleus is composed of rather large (tens of centimeters to hundreds of meters) porous refractory boulders "cemented" together with an ice-dust mixture $/ 5 /$.

The chemical composition and physical structure of the active surface layers are very important factors affecting the mass, momentum and energy densities of the outflowing gas-dust mixture, as well as the relative abundance of various gas molecules. The prevailing view is that the solid components of cometary active regions form an extremely porous, low density, weak structure, rather than a coherent mass of rocky solids penetrated by gas or liquids that froze (cf. /4-6/). When the nucleus approaches the sun, it absorbs an increasingly larger flux of solar radiation thus increasing the vaporization rate of volatile molecules at the surface. The vaporized gases leave the surface and form an expanding exosphere. In this process the gas drags away some of those dust grains which have already been evacuated of their ice component, while a fraction of these dust grains may form an inert insulating layer (mantle) over the active surface. The development of such a mantle was modeled by Mendis and Brin $/ 7 /$, Brin and Mendis $/ 8 /$, and subsequently by Horanyi et al. 19/, Fanale and Salvail /10/, Podolak and Herman /11/, and Houpis et al. /12,13/. The thickness of the mantle varies with time because the continuous vaporization increases the thickness of the evacuated layer, and the "erosion" due to the drag of the outflowing gas decreases it. A schematic representation of such a mantle is shown in Figure 1.

At the surface the absorbed radiation energy is balanced by black body reradiation and downward heat conduction. Inside the mantle a small fraction of the downward conducted energy heats the outward diffusing gas, while most of the available heat is transported to the core/mantle interface, where the vaporization process takes place. Using the mantle thickness as a free parameter one can determine the surface and mantle/core interface temperatures together with the gas production rate by solving the energy balance and heat conduction equations (cf $/ 2 /)$ : 


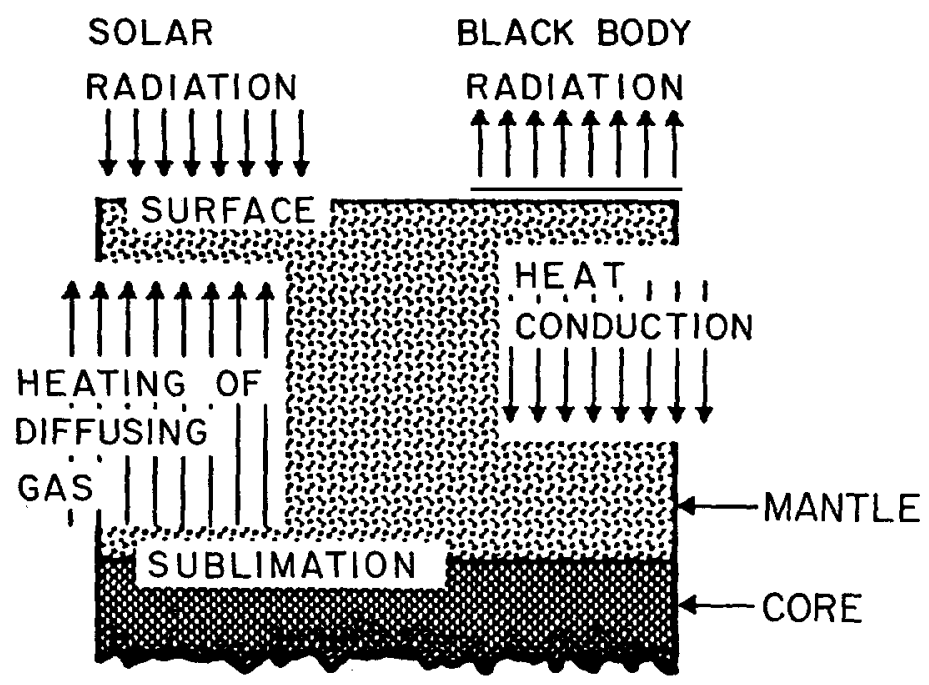

Fig.1. Schematic representation of energy transfer in a cometary mantle.
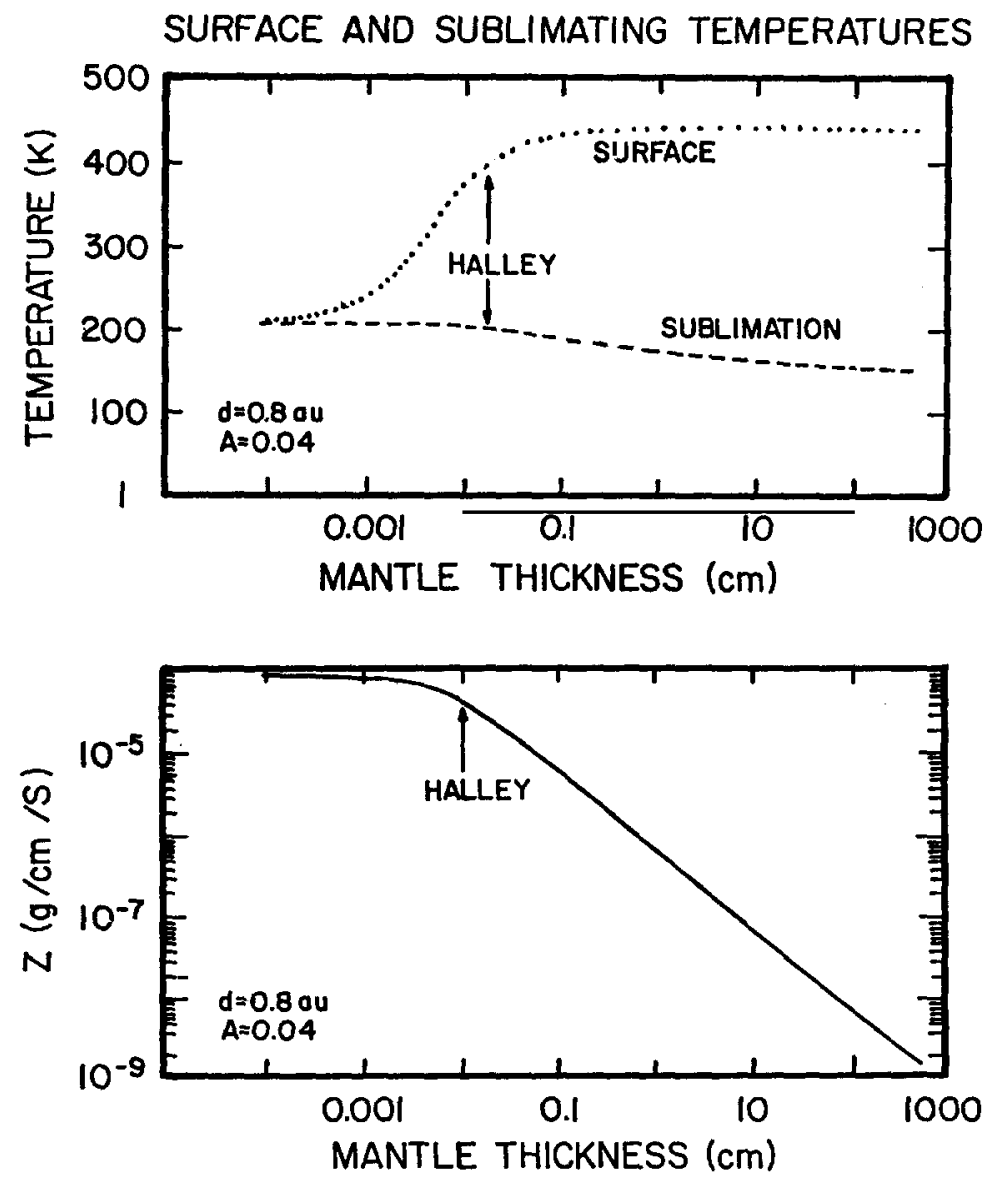

Fig. 2. Surface and sublimating temperature (upper panel) and active region gas production rate (lower panel) values as a function of mantle thickness. 


$$
\begin{aligned}
& (1-A) I_{\mathrm{rad}}-\varepsilon \sigma T_{0}^{4}=B\left(T_{L}+T_{0}-T_{1}\right) \\
& B \Delta / K_{0}=\ln \left[1+\left(T_{0}-T_{1}\right) / T_{L}\right]
\end{aligned}
$$

where $A=$ surface albedo, $\varepsilon=$ surface emissivity, $\sigma=$ Stefan-Boltzmann constant $\left(\sigma=5.67 \times 10^{-5} \mathrm{erg} \mathrm{cm}^{-2} \mathrm{~s}^{-1} \mathrm{~K}^{-4}\right)$, $I_{\text {rad }}=$ radiation energy flux, $\Delta=$ mantle thickness, $\kappa_{0}=$ mantle heat conductivity (a value of $k_{0}=60 \mathrm{erg} \mathrm{cm}^{-1} \mathrm{~s}^{-1} \mathrm{~K}^{-1}$
was adopted $/ 14 /), T_{0}=$ surface temperature, $T_{1}=$ mantle/core interface temperature. The $T_{L}$ and $B$ quantities are defined as

$$
\mathrm{T}_{\mathrm{L}}=\frac{2 \mathrm{~L}}{3 \mathrm{k} \mathrm{N}} \quad \mathrm{B}=1.5 \mathrm{k \eta z} / \mathrm{m}
$$

where $\mathrm{L}=$ latent heat of vaporization per mole, $\mathrm{k}=$ Boltzmann constant $\left(\mathrm{k}=1.38 \times 10^{-16} \mathrm{erg} \mathrm{K} \mathrm{K}^{-1}\right), \mathrm{N}_{\mathrm{A}}=$ Avogadro's constant $\left(\mathrm{N}_{\mathrm{A}}=6.022 \times 10^{23}\right.$ molecules $\left./ \mathrm{mole}\right), \mathrm{m}=$ mass of the volatile molecule, $\eta=$ area fraction of ice at the mantle/core interface. In a first approximation the gas mass production rate, $z$, can be expressed as

$$
z=z_{0} \frac{\exp \left(-3 T_{L} / 2 T_{1}\right)}{T_{0}^{0.5}}(1-0.062 x)
$$

where $\chi=$ dust $/$ gas mass production rate ratio (for comet Halley $\chi=0.3 / 15,16 /$ ). For a water dominated comet $\mathrm{z}_{0}=$ $9.65 \times 10^{8} \mathrm{~g} \mathrm{~K}^{0.5} \mathrm{~cm}^{-2} \mathrm{~s}^{-1}$, and $\mathrm{T}_{\mathrm{L}}=3851 \mathrm{~K}$.

The results for a heliocentric distance of $0.8 \mathrm{AU}$ (Halley encounter distance) are shown in Figure 2. The radiation energy density at the nucleus was assumed to be that of the unattenuated solar radiation $/ 1 /$, the surface albedo was assumed to be $0.04 / 17,18 /$, and a value of $8 \times 10^{6} \mathrm{erg} / \mathrm{g} / \mathrm{K}$ was adopted for the dust specific heat $/ 14 /$. Inspection of Figure 2 reveals that a mantle thickness of about $1 \mathrm{~cm}$ increases the surface temperature well above $400 \mathrm{~K}$, thus drastically reducing the fraction of the absorbed radiation energy available for supporting vaporization (almost all absorbed energy is reradiated by black body radiation).

At the time of the VEGA-1 encounter comet Halley had a total gas production rate of about $10^{30} \mathrm{molecules} / \mathrm{sec} / 15 /$ and an active area of about $50 \mathrm{~km}^{2} / 18 /$; consequently, the average gas production rate of the active region was about $6 \times 10^{-5} \mathrm{~g} / \mathrm{cm}^{2} / \mathrm{sec}$. This value indicates that the evacuated dust layer covering the active area is typically $0.01 \mathrm{~cm}$ thick. This mantle thickness predicts a surface temperature around $400 \mathrm{~K}$, which is in good agreement with the VEGA infrared observations indicating an average surface temperature well in excess of $300 \mathrm{~K} / 19 \%$.

\section{GAS AND DUST FLOW}

It was recognized as early as the mid 1930's that gas outflow plays an important role in cometary dust production $/ 20 \%$. As the vaporized gases leave the surface they drag away some of those dust grains which have already been evacuated of their ice component. The gas drag force accelerates the dust particles to terminal velocities comparable with the gas flow velocity. In early treatments of the gas-dust interaction it was assumed that the dust drag coefficient was independent of the gas parameters and that the gas velocity was constant in the dust acceleration region.

A two component treatment of the gas-dust interaction was published by Probstein in the late 1960 's $/ 21 /$. Probstein made the assumption that the heavy dust grains have no random motion and collide only with gas molecules. He also pointed out that the gas mean free path was much larger than dimensions of the dust particles, consequently the gas flow could be considered to be free molecular relative to the dust component.

Even though both the mathematical formulation and the methods of solution of the gas-dust interaction problem have been improved significantly since Probstein's original work, cometary dusty gas flow calculations are still based on the Probstein approach $22-29 \%$. In these models the mass, momentum and energy conservation equations of the single fluid neutral gas are the following:

$$
\begin{aligned}
& \frac{D \rho}{D t}+\rho \operatorname{div}(\underline{\underline{L}})=0 \\
& \rho \frac{\mathrm{Du}}{\mathrm{Dt}}+\operatorname{grad}(\mathrm{p})-\rho \underline{G}=-\mathrm{E}_{\mathrm{gd}} \\
& \frac{D}{D t}\left(\frac{1}{\gamma-1} p\right)+\left(\frac{\gamma}{\gamma-1} p\right) \operatorname{div}(\underline{\mathrm{l}})+\operatorname{div}(\underline{q})=Q_{\mathrm{ext}}-Q_{g d}
\end{aligned}
$$

where $\rho=$ mass density, $p=$ gas pressure, $\underline{u}=$ gas velocity, $\underline{q}=$ heat flux, $E_{g d}=$ gas to dust momentum transfer rate, $\mathrm{Q}_{\mathrm{gd}}=$ gas to dust energy transfer rate, $Q_{\mathrm{ext}}=$ external heating rate. The equation of motion of an individual dust grain 


$$
\frac{\mathrm{d} \underline{\mathrm{V}}_{\mathrm{a}}}{\mathrm{dt}}=\frac{3}{4 a \rho_{\mathrm{a}}} p \mathrm{C}_{\mathrm{D}} \underline{\mathrm{s}}_{\mathrm{a}}+\underline{G}
$$

where $\mathrm{a}=$ dust particle radius, $\underline{\mathrm{V}}_{\mathrm{a}}=$ dust particle velocity, $\rho_{\mathrm{a}}=$ dust grain bulk density. The dimensionless gas-dust relative velocity, $\underline{s}_{\mathrm{a}}$, and the moditied drag coefficient are

$$
\begin{aligned}
& \underline{s}_{a}=\frac{\underline{u}-\underline{V}_{a}}{(2 \mathrm{RT})^{0.5}} \\
& \mathrm{C}_{D}=\frac{2 \pi^{0.5} \mathrm{~T}_{\mathrm{a}}^{0.5}}{3 \mathrm{~T}^{0.5}}+\frac{2 \mathrm{~s}_{\mathrm{a}}{ }^{2}+1}{\pi^{0.5} \mathrm{~s}_{\mathrm{a}}{ }^{2}} \exp \left(-\mathrm{s}_{\mathrm{a}}{ }^{2}\right)+\frac{4 \mathrm{~s}_{\mathrm{a}}{ }^{4}+4 \mathrm{~s}_{\mathrm{a}}{ }^{2}-1}{2 \mathrm{~s}_{\mathrm{a}}{ }^{3}} \operatorname{erf}\left(\mathrm{s}_{\mathrm{a}}\right)
\end{aligned}
$$

where $R=k / m, T=$ gas temperature, $T_{a}=$ dust grain temperature, and $s_{a}=\mid \underline{s}_{a} l$. In the presence of an external radiation field the energy balance equation for a single dust particle is:

$$
C_{a} \frac{d T_{a}}{d t}=\frac{3}{a \rho_{a}}\left(p T^{0.5} C_{H}+0.25 \varepsilon_{a b s} I_{\text {rad }}-\varepsilon_{\text {emiss }} \sigma T_{a}{ }^{4}\right)
$$

where $C_{\mathrm{a}}=$ dust specific heat, $\varepsilon_{\mathrm{abs}}$ and $\varepsilon_{\mathrm{emiss}}$ are the dust absorption and infrared reemission emissivities, respectively, while

$$
C_{H}=\frac{(2 R)^{0.5}}{(\gamma-1)} \Gamma_{s}\left(2 \gamma+2(\gamma-1) s_{a}^{2}-\frac{(\gamma-1) \operatorname{ert}\left(s_{a}\right)}{s_{a} \Gamma_{s}}-(\gamma+1) \frac{T_{a}}{T}\right)
$$

where

$$
\Gamma_{\mathrm{s}}=\pi^{-0.5} \exp \left(-\mathrm{s}_{\mathrm{a}}{ }^{2}\right)+\left(0.5 / \mathrm{s}_{\mathrm{a}}+\mathrm{s}_{\mathrm{a}}\right) \operatorname{erf}\left(\mathrm{s}_{\mathrm{a}}\right)
$$

Finally, the dust size distribution function, $f_{a}$, must obey the following continuity equation:

$$
\frac{\partial_{a}}{\partial t}+\operatorname{div}\left(f_{a} \underline{V}_{a}\right)=0
$$

The gas to dust momentum and energy transfer rates can be obtained by integrating over all dust sizes:

$$
\begin{aligned}
& E_{g d}=p \int_{a_{0}}^{a_{m}} d a 2 \pi a f_{a} \underline{s}_{a} C_{D} \\
& Q_{g d}=p \int_{a_{0}}^{a_{m}} d a 2 \pi a f_{a}\left(\underline{V}_{a} \underline{s}_{a} C_{D}+4 T^{0.5} C_{H}\right)
\end{aligned}
$$

where $a_{0}$ and $a_{m}$ represent the minimum and maximum dust sizes. External gas heating is mainly caused by

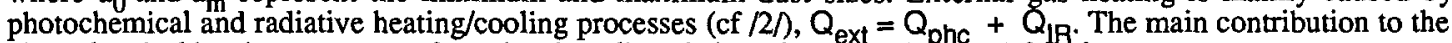
photochemical heating rate comes from the photodissociation of water molecules ( $\mathrm{cf} / 3 \mathrm{l} /$ ):

$$
Q_{p h c}=Q_{0} \frac{n}{d} \exp \left(-\tau_{U V}\right)
$$

where $\mathrm{n}=\rho / \mathrm{m},=2.8 \times 10^{-17} \mathrm{erg} \mathrm{cm}^{-3} \mathrm{~s}^{-1}, \mathrm{~d}=$ heliocentric distance $(A U), \tau_{U Y}=$ ultraviolet optical depth. Two main processes contributing to the infrared radiative heating/cooling term are the infrared radiation from the $\mathrm{H}_{2} \mathrm{O}$ molecules $132,33 /$ and the radiative trapping of the dust thermal radiation $/ 29 /$ The combined effect of these processes can be approximated as (cf /2/):

$$
Q_{I R}=-Q_{\text {emiss }} n \exp \left(-\tau_{I R}\right)+h_{I R} q_{a b s} \sigma\left[1-\exp \left(-\tau_{I R}\right)\right] \int_{a_{0}}^{a_{m}} d a a^{2} f_{a} T_{a}^{4}
$$


where $\sigma=$ Stefan-Boltzmann constant $\left(\sigma=5.67 \times 10^{-5} \mathrm{erg} \mathrm{cm}^{-2} \mathrm{sec}^{-1} \mathrm{~K}^{-4}\right), h_{\mathrm{IR}}=$ infrared trapping efficiency $\left(h_{\mathrm{R}} \approx 1\right.$ ), $q_{a b s}=$ relative width of absorbing bands with respect of the infrared spectrum emitted by dust $\left(q_{a b s} \approx 0.001\right)$, and

$$
Q_{\text {emiss }}= \begin{cases}4.4 \times 10^{-22} \mathrm{~T}^{3.35} & T<52 \mathrm{~K} \\ 2.0 \times 10^{-20} \mathrm{~T}^{2.47} & T \geq 52 \mathrm{~K}\end{cases}
$$

In a spherically symmetric, steady-state case the gas continuity, momentum, and energy equations can be combined to yield the following first order differential equation for the gas velocity:

$$
\frac{d u}{d r}=\frac{u}{M^{2}-1}\left(\frac{2}{r^{2}}-\frac{F_{g d}}{p}+\frac{(\gamma+1)\left(Q_{g d}-Q_{e x t}\right)}{\gamma u p}\right)
$$

where $\mathbf{M}=$ gas Mach number, $A=$ area function $\left(A-r^{2}\right)$, and $A^{\prime}=$ spatial derivative of the area function. Equation (17) is a solar wind type equation, which has a singularity at the sonic point $(M=1)$. Figure 3 shows the various classes of mathematically possible solutions to equation (17). Inspection of Figure 3 reveals that most solution classes are non-physical; physical solutions must be single value functions of the cometocentric distance and must have an outflow Mach number of $M \leq 1$. It can be easily seen that there are only two potentially physical solutions. One,the comet "breeze" starts subsonically, reaches a maximum Mach number value of $M<1$, and then turns back and decreases towards an asymptotic value of $M=0$. It can be shown that this solution will provide a finite gas pressure in infinity, which makes this solution unphysical, too. The other solution which is the only physical solution, is the transonic comet "wind". The comet wind starts subsonically at the nucleus, goes through the singular sonic point, and then accelerates further.

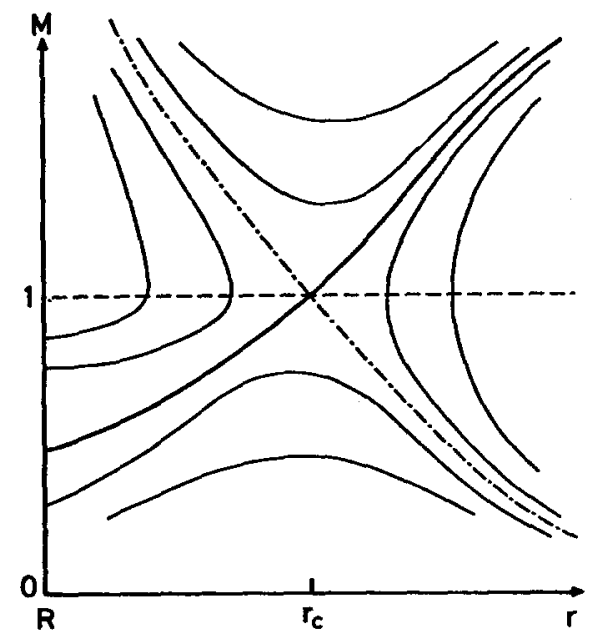

Fig. 3. Possible types of solution for equation (17). The only physical solution is the transonic "comet wind".

It is a little surprising that the physical solution of equation (17) is a transonic accelerating gas flow. At first it may not be obvious which is the external effect "accelerating" the gas and enabling it to become supersonic. In order to visualize this effect let us consider first the limiting case, when there is no dust in the system $\left(\underline{F}_{g d}=0, Q_{g d}=0\right)$ and we neglect the external heat sources, too $\left(Q_{e x t}=0\right)$. This is equivalent to seeding at the source an already established steady, radial, isentropic inviscid compressible source flow. In this case the gas flow properties can be obtained from the well known solution for a supersonic source in which the Mach number at the nucleus (source) surface is taken to be 1 in order to satisfy the appropriate boundary conditions at infinity and at the source. In other words the solution describes a free unrestricted discharge of a reservoir (containing stationary gas) to vacuum. The situation changes dramatically when the outflowing gas has had a chance to drag away dust. In this case the value of the terms inside the parenthesis in equation (17) first decrease then reach the minimum value at the sonic point (and in effect creating a $0 / 0$ type singularity) and then increase. This shows that the dust interaction (the dominant external effect near the nucleus) can be visualized using the concept of a Laval nozzle: first the outflow geometry "narrows", and then "opens up". In short, the presence of dust forces the gas flow to start at the nucleus with subsonic velocity, thus actually decelerating it. The sonic transition would have been impossible without the presence of dust. The situation strongly resembles the steady-state solar wind equation, first solved by Parker $134 /$. The gravitational effect of the sun for the solar wind corresponds to frictional forces between the dust and gas for the outflowing cometary gas. 

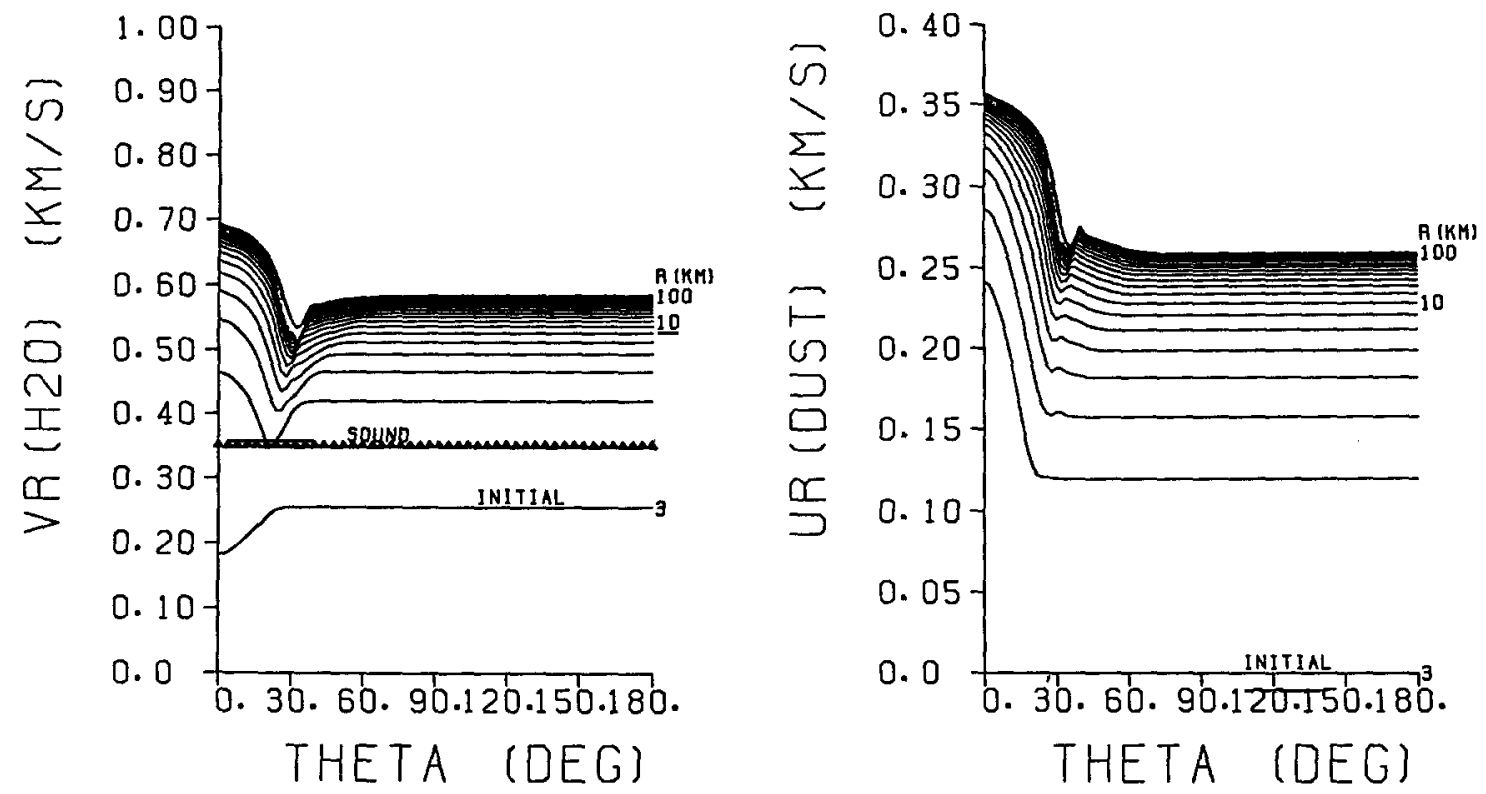

Fig. 4. Gas and dust radial velocities as a function of azimuthal angle in an axisymmetric jet model /31/.
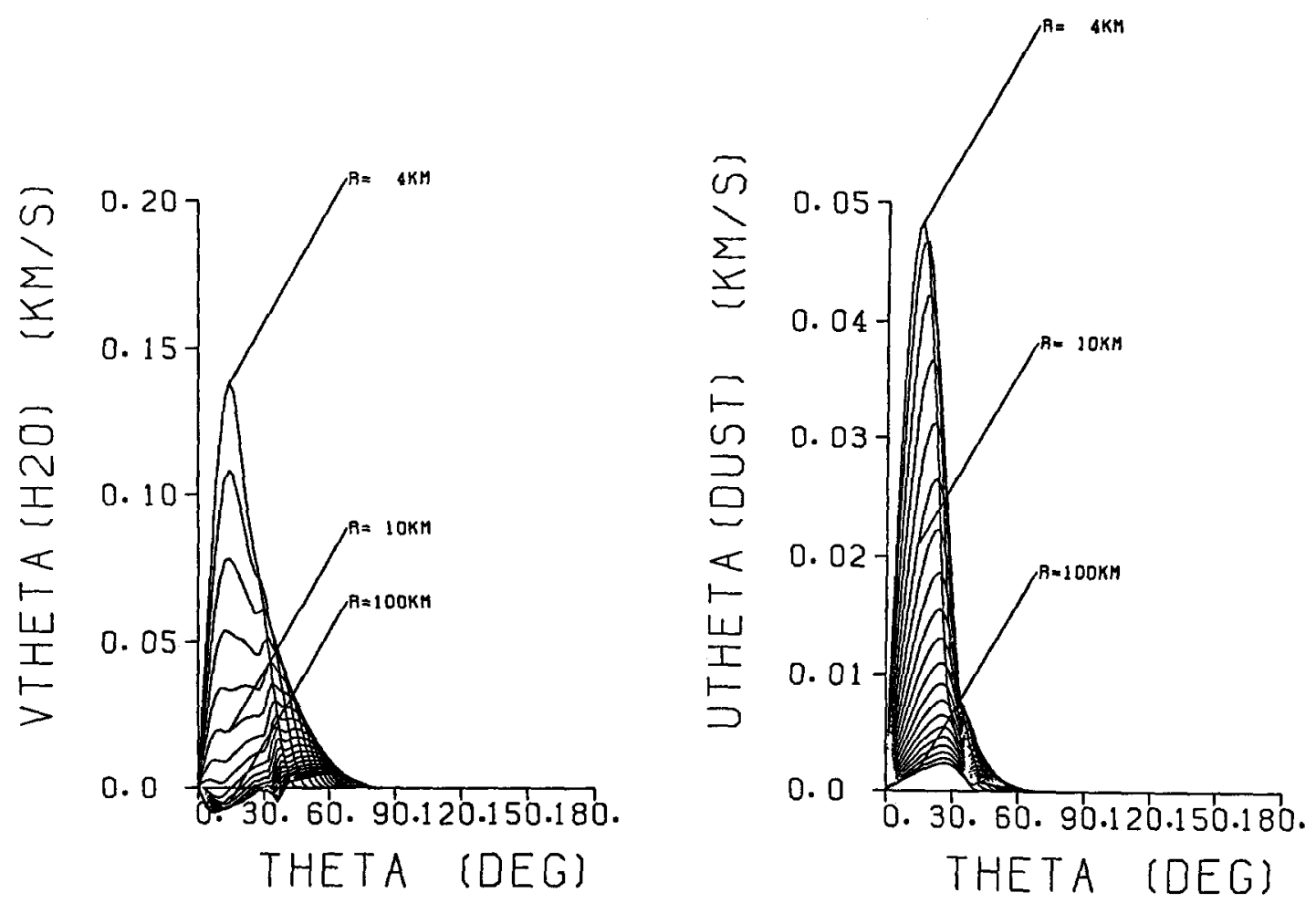

Fig. 5. Gas and dust azimuthal velocities as a function of azimuthal angle in an axisymmetric jet model $/ 31 /$. 

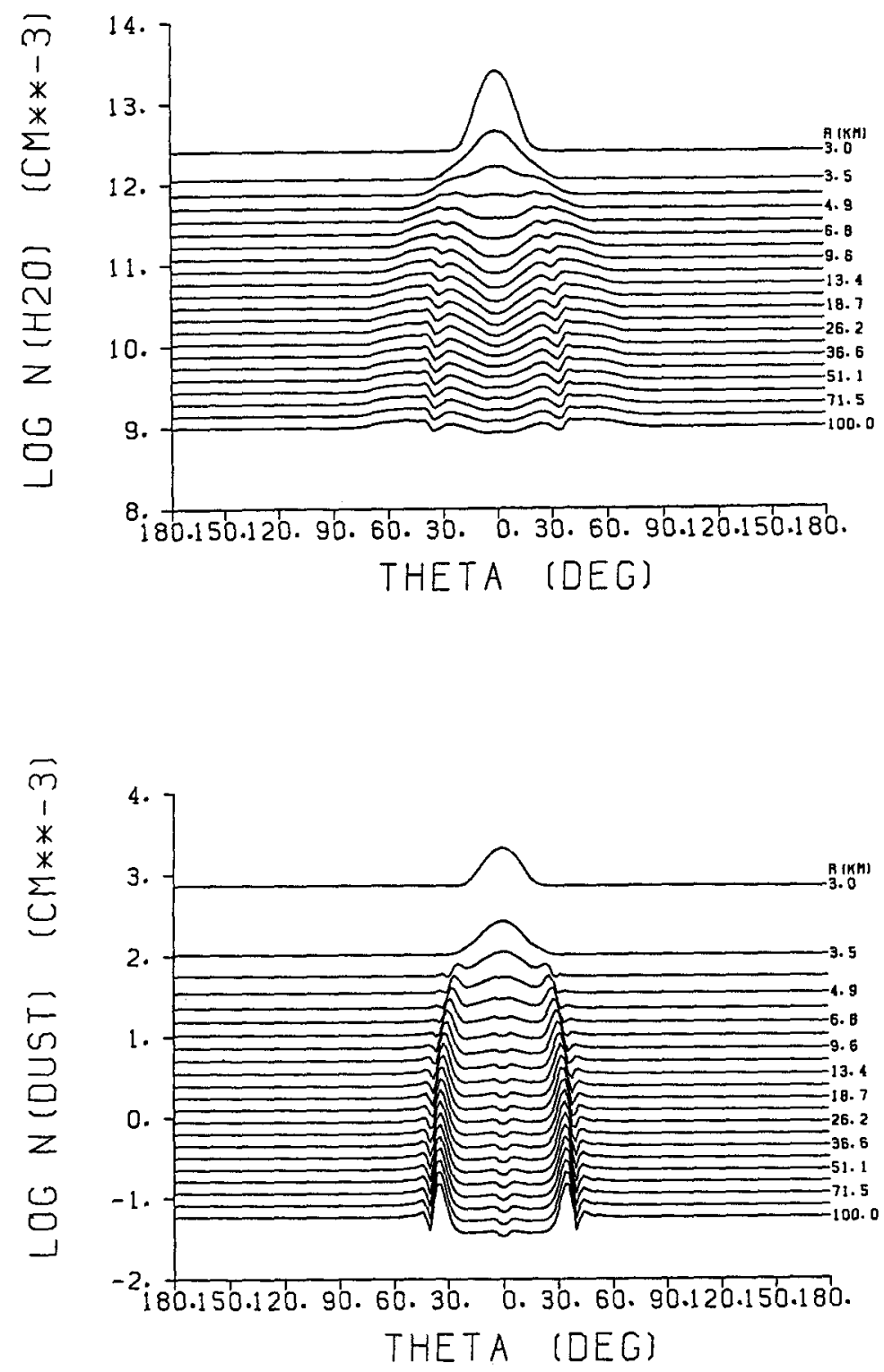

Fig. 6. Gas and dust densities as a function of azimuthal angle in an axisymmetric jet model $/ 31 /$.

Since the original work of Probstein /20/ several solutions were published to equations (4) -(14) using different levels of approximations /22-29/. Recently Kitamura /31/ published the first time-dependent axisymmetric gas jet calculation describing inner coma gas and dust distributions following a long lasting, spatially localized comet outburst. The jet profile at the surface was approximated by a Gaussian function with a half width of $10^{\circ}$. The main feature of Kitamura's numerical results is that the narrow outburst results in a conical jet. The physical reason for this conical jet formation is twofold: first of all the horizontal gas pressure gradient at the surface initiates a lateral gas transport which quickly pushes the gas density peak to $\sim 30^{\circ}$, secondly, near the nucleus the dust grains attain a tangential velocity which is comparable to their radial velocities, thus depleting the dust population along the $\Theta=0^{\circ}$ line. Further away from the nucleus the gas and dust particles lose most of their tangential velocities, consequently the modified dust structure "freezes" at a cometocentric distance of about $10 \mathrm{~km}$. The result of these two processes is a laterally varying dust/gas mass ratio, resulting in different loading effects and causing different gas terminal velocities. Figures $4-6$ (taken from Kitamura $/ 31 /$ ) show these effects in more detail. The final result is that a slow, high density conical dust jet is formed around $\Theta \sim 30^{\circ}$. Inside the jet cone the dust is faster than the ambient population, consequently the dust number density is smaller. 


\section{SUMMARY}

In this paper the governing equations of accelerating dusty gas flows in inner cometary atmospheres were summarized. The first in situ observations of comet Halley have indicated that the active surface of the nucleus is covered by a thin insulating mantle. The results of the first multidimensional dusty gas dynamical calculation $31 /$ were also reviewed pointing out that this model represents a significant improvement in describing dusty gas jets, even though it has certain limitations. In the near future one can expect a new generation of dusty gas dynamic models using more realistic input parameters and distribution deduced from the VEGA, SUISEI and GIOTTO results, further improving inner coma modeling.

This work was supported by NASA grant NGR-23-005-015 and by NSF grant ATM-08753.

\section{REFERENCES}

1. D.A. Mendis, H.L.F. Houpis, and M.L. Marconi, The physics of comets, Fundamentals of Cosmic Physics, 10 , $1(1985)$

2. Gombosi T.I., Nagy A.F., and Cravens T.E., Dust and neutral gas modeling of the inner atmospheres of comets, Reviews of Geophysics, (1986)

3. Whipple F.L., A comet model I.: The acceleration of comet Encke, Astrophysical Journal 111, 375 (1950)

4. Weissman P.R., Are cometary nuclei primordial nubble piles?, Nature, 320, 242 (1986)

5. Gombosi T.I., and Houpis H.L.F., An icy-glue model of cometary nuclei, Nature, submitted, (1986)

6. Donn B., and Rahe J., The structure and origin of cometary nuclei, in: Comets ed. L.L. Wilkening, Univ. Arizona Press, Tucson 1982, p203

7. Mendis D.A., and Brin G.D., The monochromatic brightness variations of comets II.: The core-mantle model, The Moon and the Planets, 17, 359 (1977)

8. Brin G.D., and Mendis D.A., Dust release and mantle development in comets, Astrophysical Journal, 229, 402 (1979)

9. Horányi M., Gombosi T.I., Cravens T.E., Körösmezey A., Kecskeméty K., Nagy A.F., and Szegö K., The friable sponge model of cometary nuclei, Astrophysical Journal, 278, 449 (1984)

10. Fanale F.P., and Salvail J.R., An idealized short-period comet model: Surface insolation, $\mathrm{H}_{2} \mathrm{O}$ flux, dust flux and mantle evolution, Icarus, 60,476 (1984)

11. Podolak M., and Herman G., Numerical simulations of comet nuclei II.: The effect of the dust mantle, Icarus, $\underline{61}$, 267 (1985)

12. Houpis H.L.F., Ip W.H., and Mendis D.A., The chemical differentiation of the cometary nucleus: The process and its consequences, Astrophysical Journal, 295, 654 (1985)

13. Houpis H.L.F., Gombosi T.I., and Körösmezey A., Physicochemical and time evolution model of the cometary nucleus, paper presented at the XXVIth COSPAR meeting, Toulouse, France, June 30 - July 11, 1986

14. Divine N., Fechtig H., Gombosi T.I., Hanner M.S., Keller H.U., Larson S.M., Mendis D.A., Newburn R.L., Reinhard R., Sekanina Z., and Yeomans D.K., The comet Halley dust and gas environment, Space Science Reviews, 45,1 (1986)

15. Gringauz K.I., Gombosi T.I., Remizov A.P., Apáthy I., Szemerey I., Verigin M.I., Denchikova L.I., Dyachkov A.V., Keppler E., Klimenko I.N., Richter A.K., Somogyi A.J., Szegö K., Szendrö S., Tátrallyay M., Varga A., and Vladimirova G.A., First in situ plasma and neutral gas measurements at comet Halley, Nature, 321, 282 (1986)

16. Simpson J.A., Sagdeev R.Z., Tuzzolino A.J., Perkins M.A., Ksanfomality L.V., Rabinowitz D., Lentz G.A., Afonin V.V., Erö J., Keppler E., Kosorokov J., Petrova E., Szabó L., and Umlauft G., Dust counter and mass analyser (DUCMA) measurements of comet Halley's come from Vega spacecraft, Nature, 321, 278 (1986)

17. Sagdeev R.Z., Szabó F., Avanesov G.A., Cruvelier P., Szabó L., Szegö K., Abergel A., Balázs A., Barinov I.V., Bertaux J.L., Blamont J., Detaille M., Demarelis E., Dul'nev G.N., Endröczy G., Gárdos M., Kanyó M., Kostenko V.I., Krasikov V.A., Nguyen-Trong T., Nyitrai Z., Rényi I., Rusznyák P., Shamis V.A., Smith B., Sukhanov K.G., Szalai S., Tarnopolsky V.I., Tóth I., Tsukanova G., Valnicek B.I., Várhalmi L., Zaiko Yu.K., Zatsepin S.I., Ziman Ya.L., Zsenei M., and Zhukov B.S., Television observations of comet Halley from Vega spacecraft, Nature, 321,262 (1986) 
18. Keller H.U., Arpigny C., Barbieri C., Bonnet R.M., Cazes S., Coradini M., Cosmovici C.B., Delamere W.A., Huebner W.F., Hughes D.W., Jamar C., Malaise D., Reitsema H.J., Schmidt H.U., Schmidt W.K.H., Seige P., Whipple F.L., and Wilhelm K., First Halley Multicolour Camera imaging results from Giotto, Nature, $\underline{321}$, $320(1986)$

19. Combes M., Moroz V.I., Crifo J.F., Lamarre J.M., Charra J., Sanko N.F., Soufflot A., Crovisier J., Emerich C., Encrenaz T., Gispert R., Grigoryev A.V., Guyot G., Krasnopolsky V.A., Nikolsky Yu.V., and Rocard F., Infrared sounding of comet Halley from Vega 1, Nature, 321,266 (1986)

20. Orlov S.V., Komety, ONTI, Moscow, (1935)

21. Probstein R.F., The dusty gas dynamics of comet heads, in: Problems of Hydrodynamics and Continuum Mechanics, published by the Soc. Industr. Appl. Math., 1968, p568

22. Hellmich R., and Keller H.U., On the dust production rates of comets, in: J. Halliday and B.A. McIntosh, D. Reidel, Dordrecht, 1980, p255

23. Hellmich R., and Keller H.U., On the visibility of nuclei of dusty comets, Icarus, 47, 325 (1981)

24. Gombosi T.I., Szegö K., Gribov B.E., Sagdeev R.Z., Shapiro V.D., Shevchenko V.I., and Cravens T.E., Gas dynamic calculations of dust terminal velocities with realistic dust size distributions, in: Cometary Exploration (ed. T.I. Gombosi), KFKI Press, Budapest, 1983, Vol. 2, p99

25. Gombosi T.I., Cravens T.E., and Nagy A.F., Time-dependent dusty gasdynamical flow near cometary nuclei, Astrophysicsl Journal, 293, 328 (1985)

26. Marconi M.L., and Mendis D.A., A multi-fluid model model of an $\mathrm{H}_{2} \mathrm{O}$ dominated dusty cometary nucleus, The Moon and the Planets, 27, 431 (1982)

27. Marconi M.L., and Mendis D.A., The atmosphere of a dirty clathrate cometary nucleus: A two-phase multi-fluid model, Astrophysical Journal, 273, 381 (1983)

28. Marconi M.L., and Mendis D.A., The effects of the diffuse radiation fields due to multiple scattering and thermal reradiation by dust on the dynamics and thermodynamics of a dusty cometary atmosphere, Astrophysical Journal, $\underline{287}, 445(1984)$

29. Marconi M.L., and Mendis D.A., IR heating of the cometary atmosphere, Earth. Moon and Planets, submitted, (1986)

30. Huebner W.F., The photochemistry of comets, in: ed.J.S. Levine, Academic Press, New York, 1985, p438

31. Kitamura Y., Axisymmetric dusty gas jet in the inner coma of a comet, Icarus, submitted, (1986)

32. Shimizu M., Neutral temperature of cometary atmospheres, in: The Study of Comets eds.B. Donn, M. Mumma, W. Jackson, and R. Harrington, NASA S-393, 1976, p363

33. Crovisier J., The water molecule in comets: fluorescence mechanisms and thermodynamics of the inner coma, Astronomy and Astrophysics, 130, 361 (1984)

34. Parker E.N., Dynamics of the interplanetary gas and magnetic fields, Astrophysical Journal, 128,664 (1958) 